\title{
Telescopic coronary sinus cannulation for mapping and ethanol ablation of arrhythmia originating from left ventricular summit
}

\author{
Artur Baszko, Piotr Kałmucki, Tomasz Siminiak, Andrzej Szyszka \\ Poznan University of Medical Sciences, Poznan, Poland
}

\section{Introduction}

The radiofrequency ablation (RFA) of arrhythmia from left ventricular (LV) summit encounters several difficulties [1]. There are several ablative techniques used to treat LV summit arrhythmia: high energy irrigated ablation [2], ablation through the great cardiac vein [3], epicardial ablation [4] or bipolar ablation $[5,6]$. The technique which has recently gained interest is transvenous ethanol ablation [7]. As there is no generally accepted method for alcohol ablation, herein the telescopic coronary sinus (CS) cannulation technique from femoral vein was introduced.

\section{Description of the method}

The telescopic system consists of an $8 \mathrm{~F}$ SL0 catheter, a $5 \mathrm{~F}$ guide catheter and microcatheter. All procedures were performed using the FD10 Allura Xper angiography (Philips, Netherlands) and electroanatomic system Carto 3 (Biosense Webster, Inc, Diamond Bar, CA, USA) or Ensite Velocity (St. Jude Medical Inc., St. Paul, MN, USA).

\section{Coronary sinus angiography}

After preparation, the left femoral vein is punctured and two electrodes are positioned in His region (5 F 4-pole) and CS (steerable $4 \mathrm{~F} 10$ poles, Abbott). The $4 \mathrm{~F}$ electrode records the activation from the distal part of CS to confirm epicardial character of the arrhythmia [3]. The $8 \mathrm{~F} \mathrm{SL0}$ sheath (Abbott) is inserted through right femoral vein and positioned at the level of CS ostium. Access to CS is obtained with an ablation electrode (Fig. 1A). After CS intubation, a venography is performed (Fig. 1B, 2C).
For wide CS, Attain Clarity ${ }^{\mathrm{TM}} 62251$ (Medtronic, B.V, Netherlands) venography balloon catheter is optimal. It is $90 \mathrm{~cm}$ long, and the balloon diameter is $13 \mathrm{~mm}$. For smaller CS, CORODYN P1 F6 $80 \mathrm{~cm}$ (Braun, Melsungen AG, Germany) can be used with different balloon diameters ( $5 \mathrm{~F}$ : $8 \mathrm{~mm}, 6 \mathrm{~F}$ : $10 \mathrm{~mm}, 7 \mathrm{~F}: 12 \mathrm{~mm}$ ). The venography is routinely performed in several projections.

Intubation and mapping of small

venous branches

After angiography is completed, the $5 \mathrm{~F}$ guide catheter (JR4 or IMA) is introduced through SL0 sheath. The guide catheter is a better than diagnostic tool as it has shorter, soft and non-tapered distal tip. The 0.014" BMW wire (Abbot Vascular, Diegam, Belgium) is advanced to the distal part of catheter. At this stage the microcatheter is introduced over the wire (Finecross, Terumo, Tokyo, Japan). The microcatheter has $1.8 \mathrm{~F}(0.6 \mathrm{~mm})$ radiopaque distal tip. After the vein of interest is wired, the microcatheter is advanced to cover most of the wire leaving the distal $0.5 \mathrm{~cm}$ part for recording the unipolar signal (Fig. $1 \mathrm{C}, 2 \mathrm{C}$ ). The recording of intracardiac signals and pacing is possible after connecting the percutaneous coronary intervention (PCI) wire in unipolar mode with electrophysiological system. At this stage the wire is removed and a small amount of contrast is given to confirm that the correct vein has been selected, to assess the diameter of the vein and the presence of collaterals, which can be responsible for irregular distribution of ethanol and its wash up (Fig. 2E). Thereafter the guidewire is inserted again and microcatheter is replaced for over-the-wire (OTW) balloon. As the Finecross microcatheter is shorter

Address for correspondence: Artur Baszko, MD, PhD, $2^{\text {nd }}$ Department of Cardiology, Poznan University of Medical Sciences, ul. 28 Czerwca 1956 r. No. 194, 61-485 Poznań, Poland, tel: +48 61 2274164, e-mail: abaszko@wp.pl

Received: 21.11.2018

Accepted: 3.05.2019 


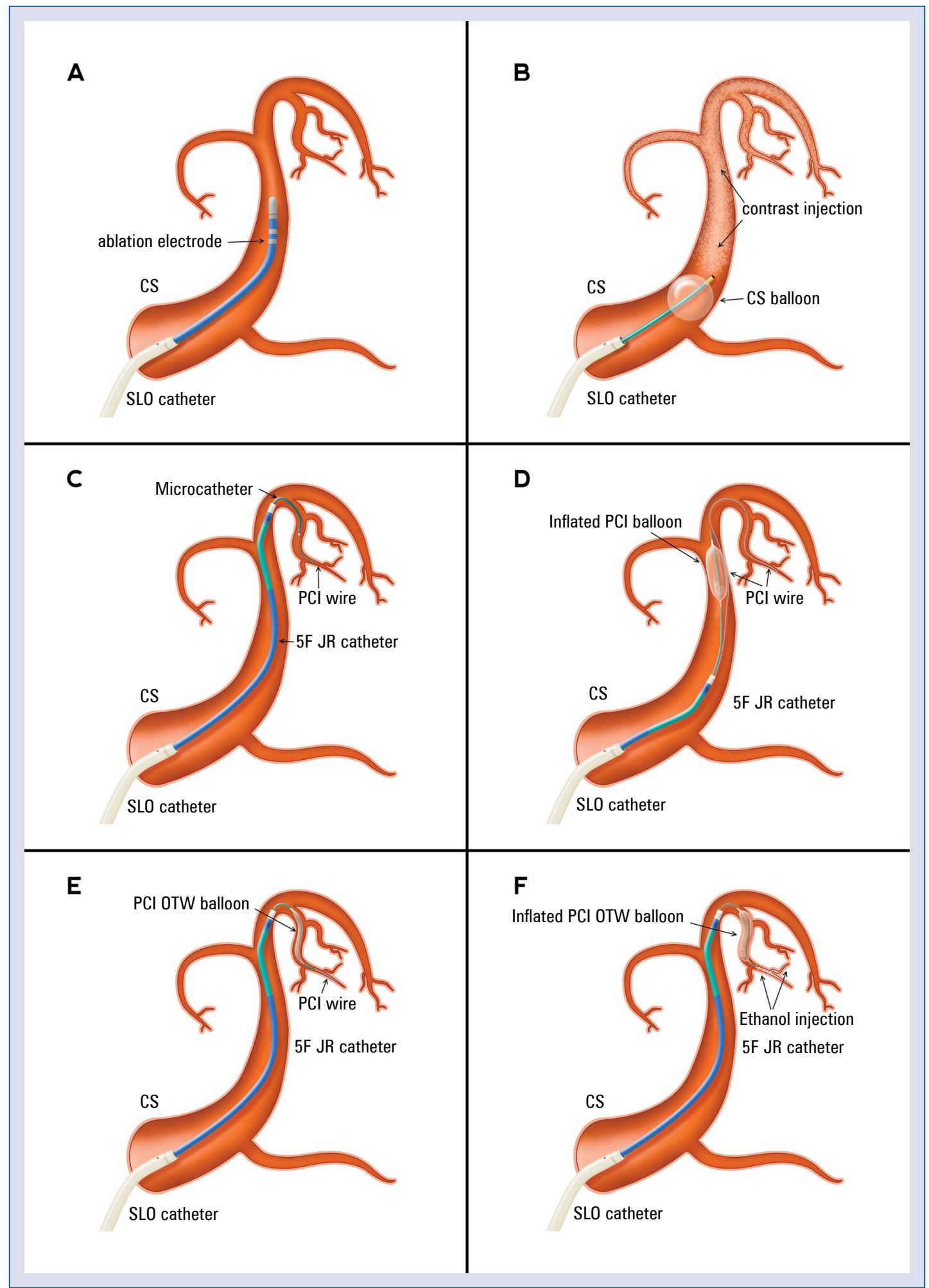

Figure 1. The crucial steps in performing transvenous ethanol ablation of arrhythmia with telescopic system; A. The intubation of the coronary sinus (CS) with ablation electrode and 8.5 F SL0 sheath; B. Coronary sinus venography through SL0 sheath; C. The 5 F JR guide catheter and angioplasty insertion through SL0 catheter followed by a microcatheter. The mapping is performed connecting the proximal percutaneous coronary intervention (PCI) wire with electrophysiological system in unipolar mode (not presented in the picture). The contrast can be selectively administered to the small branch through a microcatheter after the $\mathrm{PCl}$ wire is removed; $\mathbf{D}$. Replacement of a microcatheter using the "trapping technique" with angioplasty balloon; E. The contrast injection through over-the-wire (OTW) balloon; F. Ethanol injection through inflated OTW balloon according to the method described by Kreidieh et al. [8]. 

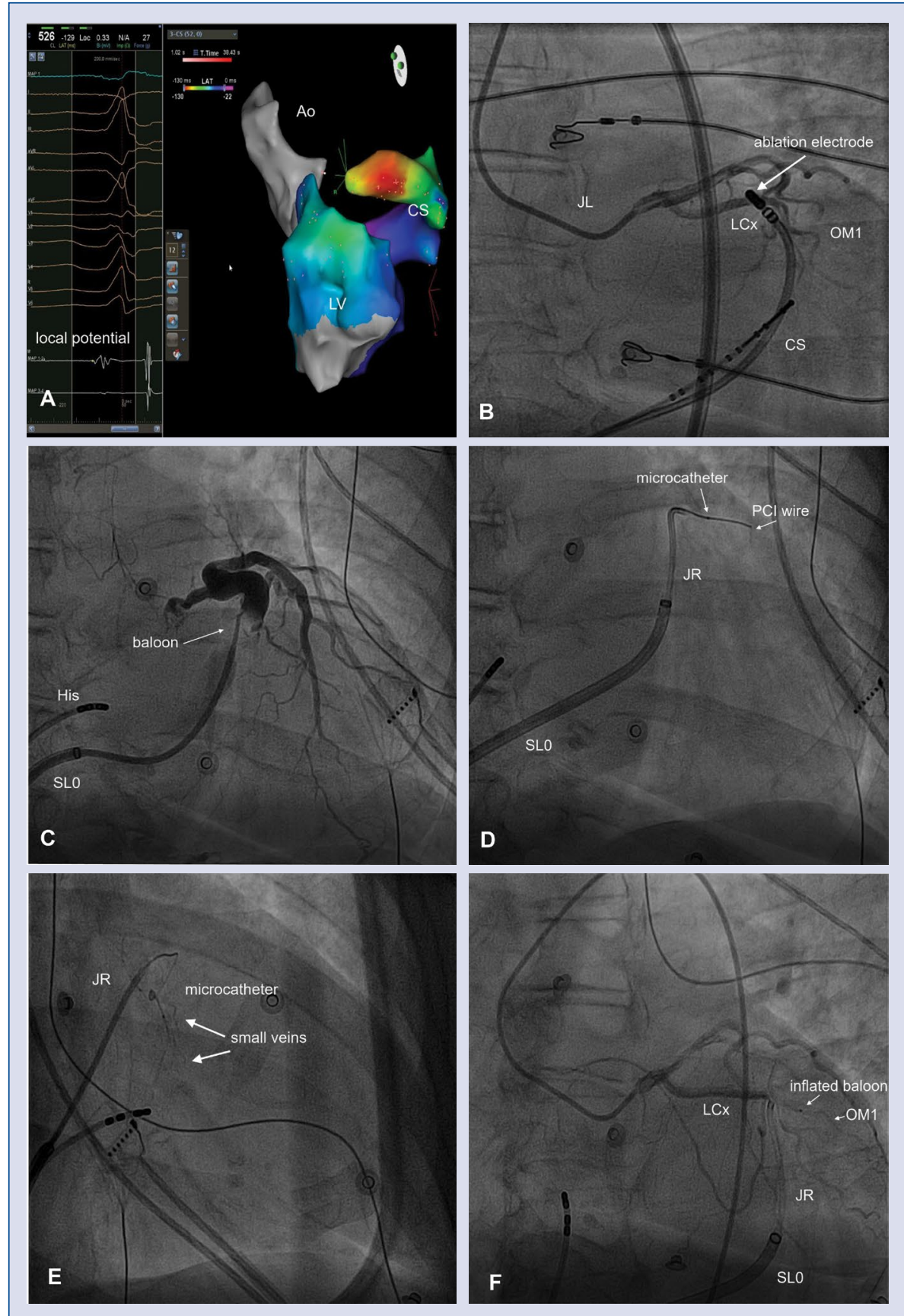

Figure 2. The important stages of ethanol transvenous ablation with telescopic coronary sinus cannulation technique after previously failed radiofrequency ablation (RFA); A. A 12-lead electrocardiogram of clinical VPC and the CARTO electroanatomical map presenting the earliest potentials within the distal part of coronary sinus (CS); B. The position of ablation electrode within CS. The "best" potential was recorded at the site of obtuse marginal branch (OM1). The RFA was not performed because of the high risk of arterial damage; $\mathbf{C}$. The CS angiography performed with the use of SL0. The angiography clearly shows several potential branches for detailed mapping; $\mathbf{D}$. The cannulation system consisted of SLO and JR catheters enabling the introduction of a microcatheter and percutaneous coronary intervention (PCl) wire. The microcatheter creates the insulation of the $\mathrm{PCl}$ wire for recording unipolar signals and pacing from the distal part of the wire; E. After selecting the optimal site for ethanol ablation, the $\mathrm{PCl}$ wire can be removed and a small amount of contrast can identify the region supplied including possible collaterals; F. Presentation of location of inflated over-the-wire (OTW) balloon before ethanol injection together with coronary angiography. This picture presents the location of the potential site for ablation position very close to OM1 branch. Delivering a radio frequency application in this region could result in injury to OM1 branch while delivering the ethanol, though the vein dose is safe. 
than the guidewire, there are two techniques for the exchange. In a "flushing technique", the inflator is connected to the microcatheter after it has been withdrawn and reaches the proximal part of the wire. A fast injection of saline with controlled pulldown ejects the wire from microcatheter leaving the distal part almost in place. In a "trapping technique", the angioplasty balloon is used to trap the angioplasty wire (Fig. 1D). The trapping of the wire can be obtained within the guide catheter or in the great cardiac vein depending on the anatomy. The standard semi compliant balloon $(1.5 \times 15 \mathrm{~mm}$ for JR or $2.5 \times 15 \mathrm{~mm}$ for CS) is inserted through the JR catheter by the side of the microcatheter and advanced to the distal part of the catheter or to the great cardiac vein passing the tip of the microcatheter. Thereafter the balloon is inflated to trap the wire well against the JR or CS wall enabling safe removal of the microcatheter. The last stage is to insert the OTW balloon to a previously selected position (Fig. 1E, 2F). The wire is then removed, the balloon is inflated and a small amount of contrast is administered to visualize the drained region (Fig. 1F). An ethanol injection can be injected according to a technique described by Kreideih et al. [8].

The technique described was evaluated prospectively in 4 patients (17-65 years, 3 females) with LV summit ventricular tachycardia after 1-3 failed RFA. The CS was easily cannulated without complications in all patients and there were no technique related problems. The time for CS cannulation was $2-5 \mathrm{~min}$, the time from CS intubation to contrast injection to venous branches ranged 17-29 $\mathrm{min}$, and the time taken from selective venous angiography to first alcohol injection ranged from 28 to $31 \mathrm{~min}$. The time from CS intubation to first ethanol injection ranged from 66 to $86 \mathrm{~min}$. The mean fluoroscopy time of the procedure was $52 \mathrm{~min}$ (range 32-86 min) and the Air-Kerma dose $422 \mathrm{mGy}$ (range 45-818 mGy) which included all stages of the procedure. The telescopic system was easy to control, allowed for rapid electrodes and catheter replacement. No obstacles or complications were noted during mapping or ablation therapy.

The described technique for transvenous ethanol ablation using $8 \mathrm{~F} \mathrm{SL} 0$ catheter with $5 \mathrm{~F}$ guide catheter and microcatheter from the femoral vein is comfortable for both the patient and the cardiologist. All three components are important at each consecutive step of the procedure. The SL0 catheter helps to obtain safe and stable access to the CS with the ablation electrode and the balloon for performing angiography. The routine use of microcatheters is definitely more expensive than OTW balloons, but there are several benefits. They help exchange the angioplasty wire when the distal part has been deformed or a different wire or tip shape is required. The use of a microcatheter can reduce the number of OTW balloons and enables better unipolar mapping and allows deeper engagement of the wire for better support during OTW balloon insertion. It is possible that a routine use of femoral access with microcatheters for mapping and identification of the target site can be beneficial in time reduction, but this needs to be further investigated.

\section{Conflict of interest: None declared}

\section{References}

1. Yamada T, Yoshida N, Doppalapudi H, et al. Efficacy of an anatomical approach in radiofrequency catheter ablation of idiopathic ventricular arrhythmias originating from the left ventricular outflow tract. Circ Arrhythm Electrophysiol. 2017; 10(5): e004959, doi: 10.1161/CIRCEP.116.004959, indexed in Pubmed: 28500177.

2. Everett TH, Lee KW, Wilson EE, et al. Safety profiles and lesion size of different radiofrequency ablation technologies: a comparison of large tip, open and closed irrigation catheters. J Cardiovasc Electrophysiol. 2009; 20(3): 325-335, doi: 10.1111/j.15408167.2008.01305.x, indexed in Pubmed: 18803562.

3. Baman TS, Ilg KJ, Gupta SK, et al. Mapping and ablation of epicardial idiopathic ventricular arrhythmias from within the coronary venous system. Circ Arrhythm Electrophysiol. 2010; 3(3): 274-279, doi: 10.1161/CIRCEP.109.910802, indexed in Pubmed: 20400776.

4. Santangeli P, Marchlinski FE, Zado ES, et al. Percutaneous epicardial ablation of ventricular arrhythmias arising from the left ventricular summit: outcomes and electrocardiogram correlates of success. Circ Arrhythm Electrophysiol. 2015; 8(2): 337-343, doi: 10.1161/CIRCEP.114.002377, indexed in Pubmed: 25637596.

5. Baszko A, Telec W, Kałmucki P, et al. Bipolar irrigated radiofrequency ablation of resistant ventricular tachycardia with a septal intramural origin: the initial experience and a description of the method. Clin Case Rep. 2016; 4(10): 957-961, doi: 10.1002/ ccr3.648, indexed in Pubmed: 27761246.

6. Futyma P, Wysokińska A, Sander J, et al. Bipolar endo-epicardial radiofrequency ablation of arrhythmia originating from the left ventricular summit. Circ J. 2018; 82(6): 1721-1722, doi: 10.1253/ circj.CJ-17-0782, indexed in Pubmed: 29046505.

7. Baher A, Shah DJ, Valderrabano M. Coronary venous ethanol infusion for the treatment of refractory ventricular tachycardia. Heart Rhythm. 2012; 9(10): 1637-1639, doi: 10.1016/j. hrthm.2012.06.003, indexed in Pubmed: 22683748.

8. Kreidieh B, Rodríguez-Mañero M, Schurmann P, et al. Retrograde coronary venous ethanol infusion for ablation of refractory ventricular tachycardia. Circ Arrhythm Electrophysiol. 2016; 9(7): e004352, doi: 10.1161/CIRCEP.116.004352, indexed in Pubmed: 27406606 .

9. Yamada T, Doppalapudi H, Maddox WR, et al. Prevalence and Electrocardiographic and Electrophysiological Characteristics of Idiopathic Ventricular Arrhythmias Originating From Intramural Foci in the Left Ventricular Outflow Tract. Circ Arrhythm Electrophysiol. 2016; 9(9): e004079, doi: 10.1161/CIRCEP.116.004079, indexed in Pubmed: 27625170.

10. Chen W, Yao Y, Zhang S, et al. Comparison of operator radiation exposure during coronary sinus catheter placement via the femoral or jugular vein approach. Europace. 2011; 13(4): 539-542, doi: 10.1093/europace/euq515, indexed in Pubmed: 21252193. 